Published in final edited form as:

Atherosclerosis. 2015 August ; 241(2): 364-370. doi:10.1016/j.atherosclerosis.2015.05.027.

\title{
The Relationship between Carotid Intima-Media Thickness and Carotid Plaque in the Northern Manhattan Study
}

\author{
Tatjana Rundek ${ }^{a, b}$, Hannah Gardener ${ }^{a}$, David Della-Morte ${ }^{a, c}$, Chuanhui Dong $^{a}$, Digna \\ Cabral $^{a}$, Eduardo Tiozzo ${ }^{a, d}$, Eugene Roberts ${ }^{a}$, Milita Crisby ${ }^{a}, e$, Kuen Chueng $^{f}$, Ryan \\ Demmer $^{g}$, Mitchell S. V. Elkind ${ }^{g, h}$, Ralph L. Sacco ${ }^{a, b}$, and Moise Desvarieux ${ }^{g, i}$ \\ aDepartment of Neurology, University of Miami, Miller School of Medicine, Miami, FL, USA. \\ bDepartment of Public Health Sciences, University of Miami, Miller School of Medicine, Miami, FL, \\ USA. \\ 'Department of Systems Medicine, School of Medicine, University of Rome 'Tor Vergata', Via \\ Montpellier 1, 00133 Rome, Italy; and IRCCS San Raffaele Pisana, Rome, Italy. \\ dDepartment of Psychiatry, University of Miami, Miller School of Medicine, Miami, FL, USA. \\ eDepartment of Neurobiology, Care Sciences and Society, Karolinskalnstitutet and Karolinska \\ University Hospital Huddinge, 14186 Stockholm, Sweden. \\ fDepartment of Biostatistics, Mailman Public School of Health, Columbia University, New York, \\ NY, USA \\ 9Department of Epidemiology, Mailman School of Public Health, Columbia University, New York, \\ NY, USA. \\ hDepartment of Neurology, College of Physicians and Surgeons, Columbia University, New York, \\ NY, USA. \\ iCentre de Recherches Epidemiologies et Biostatistique, INSERM U1153, Paris, France
}

\begin{abstract}
Objective-Carotid intima-media thickness (cIMT) and carotid plaque (CP) are proposed biomarkers of subclinical atherosclerosis associated with stroke risk. Whether cIMT and CP are distinct phenotypes or single traits at different stages of atherosclerotic development is unclear. We explored the relationship between these markers in the population-based Northern Manhattan Study.
\end{abstract}

\footnotetext{
Corresponding Authors Tatjana Rundek, MD PhD, Department of Neurology, Miller School of Medicine, University of Miami, Clinical Research Building, CRB 1348, 1120 NW 14th Street, Miami, FL 33136, USA, Tel +1 305-243-7847, Fax +1 305-243-7801, trundek@med.miami.edu, Hannah Gardener, ScD, Department of Neurology, Miller School of Medicine, University of Miami, Clinical Research Building, 1120 NW 14th Street, Miami, FL 33136, USA, Tel +1 305-243-9283, Fax +1 305-243-7801, hgardener@med.miami.edu.

Publisher's Disclaimer: This is a PDF file of an unedited manuscript that has been accepted for publication. As a service to our customers we are providing this early version of the manuscript. The manuscript will undergo copyediting, typesetting, and review of the resulting proof before it is published in its final citable form. Please note that during the production process errors may be discovered which could affect the content, and all legal disclaimers that apply to the journal pertain.
} 
Methods-We used high-resolution ultrasound and validated imaging protocols to study the cross-sectional ( $\mathrm{N}=1,788$ stroke-free participants) and prospective relationship ( $\mathrm{N}=768$ with follow-up scan; mean years between examinations=3.5) between CP and cIMT measured in plaque-free areas.

Results-The mean age was $66 \pm 9$ ( $40 \%$ male, $19 \%$ black, $17 \%$ white, $61 \%$ Hispanic). The mean baseline cIMT was $0.92 \pm 0.09 \mathrm{~mm}, 0.94 \pm 0.09 \mathrm{~mm}$ among the $58 \%$ with prevalent plaque, $0.90 \pm 0.08 \mathrm{~mm}$ among the $42 \%$ without prevalent plaque ( $\mathrm{p}<0.0001$ ). Each $0.1 \mathrm{~mm}$ increase in baseline cIMT was associated with a 1.72-fold increased odds of plaque presence (95\% CI $=1.50-1.97$ ), increased plaque thickness (effect on the median $=0.46 \mathrm{~mm}, \mathrm{p}<0.0001$ ), and increased plaque area (effect on the median $=3.45 \mathrm{~mm}^{2}, \mathrm{p}<0.0001$ ), adjusting for demographics and vascular risk factors. Elevated baseline cIMT was associated with an increased risk of new plaque in any location at follow-up, but after adjusting for demographics and vascular risk factors this association was no longer present. No association was observed in carotid segment-specific analyses.

Conclusion-Increased cIMT was associated with baseline prevalent plaque but did not predict incident plaque independent of other vascular risk factors. This finding suggests that increased cIMT is not an independent predictor of plaque development although these atherosclerotic phenotypes often coexist and share some common vascular determinants.

\section{Keywords}

carotid artery; carotid intima media thickness; carotid plaque; atherosclerosis; carotid ultrasound

\section{Introduction}

Carotid atherosclerosis plays a large role in the etiology of stroke and cardiovascular disease (CVD). B-mode carotid ultrasound has been widely used to detect subclinical carotid atherosclerosis by quantifying carotid intima-media thickness (cIMT) and carotid plaque (CP). Both cIMT and CP have been proposed surrogate imaging biomarkers of subclinical atherosclerosis [1,2] until recently, when it became increasingly clear that cIMT and CP may be genetically and biologically distinct atherosclerotic phenotypes with evidence of heterogeneous etiology $[3,4]$. In addition, carotid atherosclerotic plaque burden, defined as the two-dimensional total plaque area (TPA) or three-dimensional total plaque volume, may be a powerful non-invasive imaging tool for vascular risk estimation, and stronger predictor for future ischemic stroke (IS) than cIMT [5-8].

cIMT and CP have been associated with prevalent and incident atherosclerotic disease with variable effects [9-11]. Whether cIMT and CP are distinct phenotypes or represent a single trait at a different stage of atherosclerotic development is unclear. Recent studies have suggested that increased cIMT more likely represents adaptive changes to increased shear stress with aging and less likely atherosclerotic changes [12]. The biological mechanism by which increased arterial wall thickening initiates focal plaque formation is poorly understood. Therefore, a greater understanding of adaptive changes in the arterial wall with aging and of how these changes relate to the development of atherosclerosis in various populations is needed. 
In the current study, we sought to examine the cross-sectional and prospective relationships between cIMT and carotid plaque phenotypes in a multi-ethnic population of northern Manhattan. We hypothesized that increased cIMT was not related to presence of carotid plaque and to development of new carotid plaque over time.

\section{Material and Methods}

\section{Study Participants}

Subjects were participants in the Northern Manhattan Study (NOMAS), an ongoing, prospective, population-based study of stroke incidence and vascular risk factors, and were concurrently enrolled in the Oral Infections and Vascular Disease Epidemiology Study (INVEST). The details of the NOMAS and INVEST designs, methods and populations have been described previously $[13,14]$.

Eligible subjects were those who a) had never been diagnosed with ischemic stroke; b) were $>40$ years old; and c) resided in Northern Manhattan for $>3$ months, in a household with a telephone. Subjects were identified by random-digit dialing and interviews were conducted by trained bilingual research assistants. Subjects were recruited from the telephone sample (telephone response rate was 91\%) to have an in-person baseline interview and assessment. The enrollment response rate was $75 \%$, the overall participation rate was $69 \%$, and a total of 3,298 subjects were enrolled with an average annual contact rate of $95 \%$. Of the 3,298 subjects, ultrasound measurements of cIMT and $\mathrm{CP}$ were performed for 1,788, and of those, 768 had multiple ultrasound measurements over time as a part of INVEST [14]. NOMAS and INVEST are approved by the Institutional Review Boards of the Columbia University Medical Center and the University of Miami. All subjects signed written consent for participation.

\section{Baseline Evaluation}

Data were collected through interviews with trained bilingual research assistants in English or Spanish. Physical and neurological examinations were conducted by study neurologists. Race-ethnicity was based upon self-identification through a series of questions modeled after the US census and conforming to standard definitions outlined by Directive 15 [15]. Standardized questions were adapted from the Behavioral Risk Factor Surveillance System by the Centers for Disease Control regarding hypertension, diabetes, smoking, and cardiac conditions [16]. Blood pressure (BP) was measured with mercury sphygmomanometers and appropriately-sized cuffs. Hypertension was defined as a BP $\geq 140 / 90 \mathrm{mmHg}$ (based on the average of two measurements during one sitting), the patient's self-reported hypertension, or use of anti-hypertensive medications. Diabetes mellitus was defined by the patient's selfreported diabetes, use of insulin or oral anti-diabetic medications, or fasting glucose $\geq 126$ $\mathrm{mg} / \mathrm{dl}$. The fasting lipid profile was measured at enrollment. Body mass index (BMI) was calculated in $\mathrm{kg} / \mathrm{m}^{2}$.

\section{Carotid Ultrasound}

High-resolution B-mode ultrasound imaging (GE LogIQ 700, 9- to 13-MHz linear-array transducer) was performed by trained and certified sonographers as previously described 
[17-19]. Presence of plaque was defined as a focal wall thickening or protrusion in the lumen more than $50 \%$ greater than the surrounding thickness. Carotid plaque area $\left(\mathrm{mm}^{2}\right)$ and maximum thickness $(\mathrm{mm})$ were measured with the automated computerized edge tracking software program M'Ath (M'Ath Inc, Paris, France) [20]. TPA was defined as the sum of all plaque areas measured in any of the carotid artery segments within an individual. cIMT was measured in areas without plaque. cIMT was calculated as a composite measure of the near and the far walls of the common carotid artery (CCA) IMT, bifurcation (bif) IMT, and internal carotid artery (ICA) IMT of both sides of the neck, and examined continuously as a mean of the maximum measurements of the 12 carotid sites. We also examined cIMT in the Bifurcation and ICA exclusively and cIMT in the CCA exclusively. Likewise, we examined plaque phenotypes in the Bifurcation and ICA exclusively. Figure 1 is a representation of cIMT and carotid plaque using high-resolution B-mode ultrasound.

\section{Statistical Analysis}

The cross-sectional association between cIMT and plaque presence was examined with logistic regression models, where cIMT was the independent variable and plaque presence was the dependent variable. Due to the non-normal distribution of plaque thickness and area with a large percentage of the study population having no plaque, we used quantile regression to examine plaque thickness and area as continuous outcomes. For individuals without plaque, a value of 0 was assigned for plaque thickness and area. We chose the median $\left(50^{\text {th }}\right.$ percentile) and $75^{\text {th }}$ percentile as our outcome variables of interest. A three model sequence was constructed: univariate (model 1); adjusted for demographics (age, sex, race-ethnicity; model 2); and adjusted for demographics and systolic blood pressure, diastolic blood pressure, antihypertensive medication use, diabetes, low-density lipoprotein (LDL) cholesterol, high density lipoprotein (HDL) cholesterol, triglycerides, statin use, and BMI (model 3). A subset of the study population had data on left ventricular mass measured using transthoracic echocardiography, and a subset had data on diastolic intraluminal CCA diameter measured using M mode ultrasound with M'Ath software. Sensitivity analyses were conducted within these subsamples adding these two variables separately to model 3 .

Next, we performed segment specific analyses as secondary exploratory analyses. We examined cIMT in the Bifurcation and ICA only in relation to plaque presence, thickness, and area in these segments only. For the latter analysis the left and right sides were examined separately. We examined cIMT in the CCA only in relation to plaque presence in any location.

Next, we used logistic regression to conduct a prospective analysis of baseline cIMT as a predictor of incident plaque from baseline to follow-up carotid ultrasound. Incident plaque was new plaque found at follow up in any of the carotid segments, which was not present at baseline at the same location. We constructed the same sequence of multivariable-adjusted models, and also controlled for the time span between the baseline and follow-up ultrasound imaging in all three models. In addition, these prospective analyses were repeated in a subpopulation restricted only to individuals who did not have carotid plaque at baseline. For each analysis, we ran exploratory models to examine any potential effects of race-ethnicity by including interaction terms between baseline cIMT and race-ethnicity while controlling 
for all covariates included in model 3. We used logistic regression to conduct an analysis of the change in IMT from baseline to follow up as a predictor of incident plaque in the full prospective sample as well as in those without plaque at baseline, using the same series of models described above and adjusted for the time spam between the baseline and follow up measurements.

Lastly, we ran secondary models to examine the segment-specific association between IMT and incident plaque during follow up. We examined CCA IMT at baseline as well as change in CCA IMT from baseline to follow up in relation to incident plaque in any segment. In addition, we examined ICA and bifurcation IMT at baseline as well as change in ICA and bifurcation IMT in relation to incident plaque in the ICA and bifurcation only. For the latter analysis the left and right sides were examined separately. Each of these analyses were repeated in the subsample restricted to those without plaque at baseline, and the same sequence of three models was run as described above.

\section{Results}

The characteristics of the study population broken down by plaque presence are reported in Table 1. Among the 1,787 participants (mean age of $66 \pm 9$ years, $40 \%$ male, $61 \%$ Hispanics, $19 \%$ non-Hispanic black, and $17 \%$ non-Hispanic white), $57 \%$ had plaque $(\mathrm{N}=1026)$, and $37 \%$ had thick plaque $>1.9 \mathrm{~mm}(\mathrm{~N}=657)$. The mean of the maximum cIMT at baseline was $0.92 \pm 0.09 \mathrm{~mm}$ (range $=0.62-1.41 \mathrm{~mm}$ ). The mean cIMT among those with plaque was $0.94 \pm 0.09 \mathrm{~mm}(0.92 \pm 0.10 \mathrm{~mm}$ for left ICA and bif, $0.93 \pm 0.10 \mathrm{~mm}$ for right ICA and bif, $0.96 \pm 0.10 \mathrm{~mm}$ for the CCA), and among those without plaque was $0.90 \pm 0.08 \mathrm{~mm}$ $(0.89 \pm 0.09 \mathrm{~mm}$ for left ICA and bif, $0.89 \pm 0.09 \mathrm{~mm}$ for right ICA and bif, $0.91 \pm 0.11 \mathrm{~mm}$ for the CCA), $\mathrm{p}<0.0001$. Among those with plaque, the median plaque thickness was $1.57 \mathrm{~mm}$ (2.00 $\mathrm{mm}$ for left ICA and bif, $1.91 \mathrm{~mm}$ for right ICA and bif), and the plaque thickness at the $75^{\text {th }}$ percentile was $2.17 \mathrm{~mm}$ ( $2.42 \mathrm{~mm}$ for left ICA and bif, $2.35 \mathrm{~mm}$ for right ICA and bif). The median total plaque area (TPA) was $4.69 \mathrm{~mm}^{2}\left(8.80 \mathrm{~mm}^{2}\right.$ for left ICA and bif, 8.99 $\mathrm{mm}^{2}$ for right ICA and bif), and TPA at the $75^{\text {th }}$ percentile was $16.21 \mathrm{~mm}^{2}\left(15.13 \mathrm{~mm}^{2}\right.$ for left ICA and bif, $14.70 \mathrm{~mm}^{2}$ for right ICA and bif). Significant independent predictors of plaque presence in this population at baseline included older age, male sex, white race, hypertension, diabetes, and elevated LDL cholesterol, while significant independent predictors of cIMT were older age, male sex, diabetes, elevated LDL, and elevated BMI (data not shown).

Table 2 shows the cross-sectional association between baseline measurements of cIMT and plaque phenotypes. In all three models, cIMT was positively associated with plaque presence, plaque thickness, and TPA. Adjustment for demographics and vascular risk factors only attenuated the associations slightly. The associations remained consistent in sensitivity analyses adding left ventricular mass and diastolic CCA diameter to model 3 (data not shown). In model 3, the prevalence of plaque was increased by $72 \%$ for each $0.1 \mathrm{~mm}$ increase in cIMT. In addition, cIMT in the Bifurcation and ICA was also positively associated with plaque presence, thickness, and TPA in the Bifurcation and ICA, and cIMT in the CCA was positively associated with plaque presence, thickness, and TPA in all segments. 
The mean time-span between the two carotid measurements among those who had multiple scans was 3.5 years (range: $1.9-7.3 ; \mathrm{N}=768$ ), and $43 \%$ of participants developed a new plaque during follow-up $(\mathrm{N}=334, \mathrm{~N}=161$ new plaque in left bifurcation and ICA, $\mathrm{N}=160$ in the right bifurcation and ICA). Of the 324 participants without plaque at baseline, $40 \%$ developed a new plaque during follow-up ( $\mathrm{N}=130, \mathrm{~N}=100$ new plaque in left bifurcation and ICA, N=100 in the right bifurcation and ICA).

Table 3 shows the association between cIMT at baseline and incident plaque during followup. In the univariate model 1, elevated cIMT at baseline was associated with an increased risk of incident plaque during follow-up in the full subpopulation as well as in the group without plaque at baseline. However, after adjustment for demographics and vascular risk factors the association was no longer significant. Findings remained consistent in sensitivity analyses including left ventricular mass and diastolic CCA diameter in the fully adjusted model, conducted in subsamples with available data for these variables (data not shown). This lack of the relationship between cIMT and incident plaque was observed across all race-ethnic groups (no significant interactions were found). The mean longitudinal change in cIMT was $0.11 \pm 0.16 \mathrm{~mm}$ among all participants, and $0.09 \pm 0.15$ and $0.12 \pm 0.15 \mathrm{~mm}$ among those with and without plaque, respectively $(\mathrm{p}=0.01)$. There was a marginally statistically significant trend towards a positive association between increasing IMT from baseline to follow up and incident plaque among the full prospective study sample $(\mathrm{p}=0.06)$ while there was no apparent relationship among those without plaque at baseline.

Table 4 shows the segment-specific relationship between baseline IMT and incident plaque in the full prospective sample, and among those without plaque at baseline. CCA IMT was positively associated with developing a new plaque in any segment during follow up, but this association was attenuated and no longer significant in multivariable-adjusted models. Among all participants, the mean longitudinal change in bifurcation and ICA cIMT was $0.11 \pm 0.18 \mathrm{~mm}$ for the left side $(0.07 \pm 0.20$ and $0.11 \pm 0.18 \mathrm{~mm}$ among those with and without any plaque, respectively, $\mathrm{p}=0.01)$ and $0.11 \pm 0.18 \mathrm{~mm}$ for the right side $(0.08 \pm 0.21$ and $0.12 \pm 0.18 \mathrm{~mm}$ among those with and without any plaque, respectively, $\mathrm{p}=0.01$ ). The mean longitudinal change in CCA cIMT was $0.12 \pm 0.19 \mathrm{~mm}$ among all participants, and $0.12 \pm 0.18$ and $0.13 \pm 0.20 \mathrm{~mm}$ among those with and without plaque, respectively $(\mathrm{p}=0.23)$. Progression in CCA IMT from baseline to follow up was positively associated with the presence of incident plaque in any location in the full sample, but the association was attenuated and no longer significant in the sample restricted to those without plaque at baseline. Baseline and progression of cIMT in the left and right ICA and bifurcation did not predict incident plaque in the respective segments.

\section{Discussion}

Accumulating evidence suggests that cIMT and CP may be distinct phenotypes rather than a manifestation of the same phenotype at different stages or phases in the progression of atherosclerosis [21-23]. Though our study design did not evaluate this hypothesis directly, the results may provide some modest indirect support. We observed a positive association between cIMT and prevalent carotid plaque phenotypes in a cross-sectional analysis, but not between cIMT and incident plaque in a prospective analysis after accounting for vascular 
risk factors. cIMT and carotid plaque seem to be distinct manifestation of an arterial wall thickening process although they often coexist. Carotid plaque therefore may not be a simple result of progressive intima-media thickening, but rather a "de novo" event.

One particularly novel component to the current study was the examination of segmentspecific relationships between cIMT and plaque. CCA cIMT predicted plaque presence overall, consistent with the findings of other studies [7, 8, 11, 24]. ICA and bifurcation cIMT was predictive of plaque in those segments on the same side in cross-sectional analyses, but not in longitudinal analyses, indicating that cIMT may not directly progress to plaque.

Atherosclerotic plaque formation represents a dynamic process involving a complex cascade of inflammatory events [25] while carotid intima-media thickening may be related to adaptive hypertrophy of the media layer and not a true representation of an atherosclerotic lesion [26]. Increased cIMT may be represented by "fatty streaks" composed of foamy macrophages which have a non-raised appearance in the arterial lumen and have been shown to regress rather than to progress to raised lesions even in the presence of risk factors [7]. In contrast, "pathologic intimal thickening" is increased cIMT, which represents the earliest manifestation of progressive atherosclerosis [7] and is rich in proteoglycans and lipids, lacks smooth-muscle cells and collagen, and may rapidly transform into plaque through mechanisms not entirely understood. Ultrasonographic measurement of cIMT however cannot distinguish these two different intima-media thickening processes. The strong relationship between cIMT and plaque in our cross-sectional analysis was most likely a result of "pathological intimal thickening" present among those individuals with atherosclerotic plaque, sharing a common pathological mechanism, which was not completely explained by the presence of traditional vascular risk factors. In the prospective analyses however, this association attenuated after adjustment for vascular risk factors, suggesting a distinct mechanism leading to formation of incident plaque, rather than a continuum of the development of plaque from intima-media thickening. Distinct mechanisms underlying the development of plaque and cIMT has been further suggested by evidence from genetic studies which demonstrated that variants in genes encoding proteins implicated in pathways leading to formation of carotid atherosclerosis (eg oxidative stress, inflammation, and diabetes) were differentially associated with cIMT and carotid plaque [21-23]. This suggests that these two phenotypes of carotid atherosclerosis, even if correlated, could be under different biological and genetic control. Although the two processes-cIMT and plaque formation-may share some common mechanisms, their overlap is partial, and their predictive power of cerebrovascular disease (CVD) risk differs [6, 27-29].

The relationship between cIMT and carotid plaque has been examined previously. The European Vascular Aging (EVA) Study demonstrated a significant association between increased cIMT measured in the CCA and both the presence and severity of atherosclerotic plaque [29]. In a longitudinal EVA study [30], the odds of having CP was 2.7-fold greater in subjects with intermediate baseline cIMT values, and 3. 7-fold greater among those with the highest baseline cIMT values compared to subjects with the lowest baseline cIMT values. Interestingly, adjustments for major vascular risk factors did not modify these values. We 
did not confirm these observations. Although our study had similar duration of follow up (3.5 years in our study, 4 years in EVA), discrepant results most likely are related to the differences in characteristics of the study populations, definitions of risk factors, and definition of plaque. Several studies conducted in predominantly Caucasian populations reported cross-sectional associations between cIMT and plaque [31-33]. In addition, five longitudinal studies, with time spans from four to twelve years, have shown positive associations between cIMT, particularly in the CCA, with the development of carotid plaque [24, 30, 34-36]. Our findings regarding CCA cIMT in relation to incident plaque are not inconsistent with the latter studies. However, differences in study design, including longer follow up in other studies [34, 35], categorization of cIMT [24, 30, 34] rather than a continuous examination, as well as differences in study sample characteristics and size may explain variability in findings. The other longitudinal studies did not examine lateralspecific ICA and bif cIMT in relation to incident plaque like we did.

In the present study, an association between cIMT and plaque at baseline was present in all race-ethnic groups, but a more robust association was found in Hispanics than in blacks and whites. The Atherosclerosis Risk in Communities (ARIC) Study showed no difference between whites and blacks in site-specific prevalence of carotid plaque, as well as a positive association between plaque and cIMT at baseline in both race-ethnicities [32]. Differences in carotid geometry, vascular risk factor profiles and differential predisposition to vascular remodeling and atherosclerotic development may be among possible factors explaining these race-ethnic variations $[27,37,38]$.

Our results do not provide direct evidence to confirm the hypothesis that carotid plaque may be a biologically different atherosclerosis phenotype from cIMT, though some of our findings are consistent with this hypothsis. Although carotid plaque seems to be more strongly influenced by environmental factors [39] than cIMT, we have reported that traditional vascular risk factors explain about $21 \%$ of the variance in the total carotid plaque burden [20] and only $11 \%$ of the variance in cIMT [40]. This suggests that other unaccounted factors, both environment and genetic, play an important role in the determination of these phenotypes. The Cohorts for Heart and Aging Research in Genomic Epidemiology (CHARGE) Consortium showed different genetic loci associated with cIMT and carotid plaque [41]. Similarly, we observe no overlap in genetic variants associated with cIMT and CP in our family study [21, 22, 42-44].

Strengths of the current study include its multi-ethnic population-based design, a systematic collection of vascular risk factors, inclusion of both cross-sectional and prospective analyses, and the use of multiple plaque phenotypes for comparisons. However, our study has several limitations. Our population is an older cohort with high burden of risk factors and great proportion of carotid plaque at baseline. Our conclusions may not be generalizable to younger and healthier populations. Also, the population sample available for our crosssectional analysis was much larger than that used in our prospective analysis, limiting the power of the longitudinal analysis. Further research with multiple follow-up ultrasound measurements and starting earlier in life is needed to fully elucidate the complex temporal relationship between intima-media thickening and plaque formation, and to determine how these atherosclerotic phenotypes interact to affect stroke risk in various populations. 


\section{Acknowledgments}

Sources of Funding

This work was supported by grants from the National Institutes of Health/National Institute of Neurological Diseases and Stroke K24 NS062737 (Dr. Rundek); R37 NS 29993 (Drs. Sacco, Elkind, Rundek, Dong, Gardener, and Cabral); R01 NS 065114 (Drs. Rundek, Tiozzo, Della-Morte, Gardener, Dong, Cabral, and Sacco); R01 DE-13094 (Drs. Desvarieux, Demmer, Rundek, Sacco); and a Chair in Chronic Disease, École des Hautes Études en Santé Publique, France (Dr. Desvarieux). The funding sources had no involvement in study design; in the collection, analysis and interpretation of data; in the writing of the report; and in the decision to submit the article for publication.

\section{References}

1. Spence JD, et al. Carotid plaque area: a tool for targeting and evaluating vascular preventive therapy. Stroke. 2002; 33(12):2916-22. [PubMed: 12468791]

2. Pignoli $P$, et al. Intimal plus medial thickness of the arterial wall: a direct measurement with ultrasound imaging. Circulation. 1986; 74(6):1399-406. [PubMed: 3536154]

3. Spence JD. Measurement of intima-media thickness vs. carotid plaque: uses in patient care, genetic research and evaluation of new therapies. Int J Stroke. 2006; 1(4):216-21. [PubMed: 18706019]

4. Della-Morte D, et al. Genetics and genomics of ischemic tolerance: focus on cardiac and cerebral ischemic preconditioning. Pharmacogenomics. 2012; 13(15):1741-57. [PubMed: 23171338]

5. Gomez CR. Carotid plaque morphology and risk for stroke. Stroke. 1990; 21(1):148-51. [PubMed: 2405546]

6. Mathiesen EB, et al. Carotid plaque area and intima-media thickness in prediction of first-ever ischemic stroke: a 10-year follow-up of 6584 men and women: the Tromso Study. Stroke. 2011; 42(4):972-8. [PubMed: 21311059]

7. Wannarong T, et al. Progression of carotid plaque volume predicts cardiovascular events. Stroke. 2013; 44(7):1859-65. [PubMed: 23735956]

8. Nanayakkara ND, et al. Nonrigid registration of three-dimensional ultrasound and magnetic resonance images of the carotid arteries. Med Phys. 2009; 36(2):373-85. [PubMed: 19291976]

9. Lorenz MW, et al. Carotid intima-media thickness progression to predict cardiovascular events in the general population (the PROG-IMT collaborative project): a meta-analysis of individual participant data. Lancet. 2012; 379(9831):2053-62. [PubMed: 22541275]

10. Rundek T, Salameh MJ. Carotid plaque assessment: a bumpy road to improved risk prediction. J Am Coll Cardiol. 2010; 56(13):1069. author reply 1069-70. [PubMed: 20846614]

11. Den Ruijter HM, et al. Common carotid intima-media thickness measurements in cardiovascular risk prediction: a meta-analysis. JAMA. 2012; 308(8):796-803. [PubMed: 22910757]

12. Finn AV, Kolodgie FD, Virmani R. Correlation between carotid intimal/medial thickness and atherosclerosis: a point of view from pathology. Arterioscler Thromb Vasc Biol. 2010; 30(2):17781. [PubMed: 19679833]

13. Sacco RL, et al. Race-ethnic disparities in the impact of stroke risk factors: the northern Manhattan stroke study. Stroke. 2001; 32(8):1725-31. [PubMed: 11486097]

14. Desvarieux M, et al. Periodontal microbiota and carotid intima-media thickness: the Oral Infections and Vascular Disease Epidemiology Study (INVEST). Circulation. 2005; 111(5):57682. [PubMed: 15699278]

15. Wallman KK, Hodgdon J. Race and ethnic standards for Federal statistics and administrative reporting. Stat Report. 1977; (77-110):450-4. [PubMed: 12229771]

16. Sacco RL, et al. The protective effect of moderate alcohol consumption on ischemic stroke. JAMA. 1999; 281(1):53-60. [PubMed: 9892451]

17. Rundek T, et al. Carotid intima-media thickness is associated with allelic variants of stromelysin-1, interleukin-6, and hepatic lipase genes: the Northern Manhattan Prospective Cohort Study. Stroke. 2002; 33(5):1420-3. [PubMed: 11988625] 
18. Rundek T, et al. Endothelial dysfunction is associated with carotid plaque: a cross-sectional study from the population based Northern Manhattan Study. BMC Cardiovasc Disord. 2006; 6:35. [PubMed: 16916467]

19. Prabhakaran S, et al. Presence of calcified carotid plaque predicts vascular events: the Northern Manhattan Study. Atherosclerosis. 2007; 195(1):e197-201. [PubMed: 17482197]

20. Kuo F, et al. Traditional cardiovascular risk factors explain the minority of the variability in carotid plaque. Stroke. 2012; 43(7):1755-60. [PubMed: 22550054]

21. Dong $\mathrm{C}$, et al. Association of the sirtuin and mitochondrial uncoupling protein genes with carotid plaque. PLoS One. 2011; 6(11):e27157. [PubMed: 22087257]

22. Della-Morte D, et al. Association of the sirtuin and mitochondrial uncoupling protein genes with carotid intima-media thickness. Transl Res. 2012; 160(5):389-90. [PubMed: 22750084]

23. Al-Shali KZ, et al. Genetic variation in PPARG encoding peroxisome proliferator-activated receptor gamma associated with carotid atherosclerosis. Stroke. 2004; 35(9):2036-40. [PubMed: 15284449]

24. von Sarnowski B, et al. Common carotid intima-media thickness and framingham risk score predict incident carotid atherosclerotic plaque formation: longitudinal results from the study of health in Pomerania. Stroke. 2010; 41(10):2375-7. [PubMed: 20814002]

25. Fuster V, et al. Atherothrombosis and high-risk plaque: part I: evolving concepts. J Am Coll Cardiol. 2005; 46(6):937-54. [PubMed: 16168274]

26. Touboul PJ, et al. Mannheim intima-media thickness consensus. Cerebrovasc Dis. 2004; 18(4): 346-9. [PubMed: 15523176]

27. Rundek T, et al. Carotid plaque, a subclinical precursor of vascular events: the Northern Manhattan Study. Neurology. 2008; 70(14):1200-7. [PubMed: 18354078]

28. Nambi V, et al. Carotid intima-media thickness and presence or absence of plaque improves prediction of coronary heart disease risk: the ARIC (Atherosclerosis Risk In Communities) study. J Am Coll Cardiol. 2010; 55(15):1600-7. [PubMed: 20378078]

29. Bonithon-Kopp C, et al. Relation of intima-media thickness to atherosclerotic plaques in carotid arteries. The Vascular Aging (EVA) Study. Arterioscler Thromb Vasc Biol. 1996; 16(2):310-6. [PubMed: 8620348]

30. Zureik M, et al. Common carotid intima-media thickness predicts occurrence of carotid atherosclerotic plaques: longitudinal results from the Aging Vascular Study (EVA) study. Arterioscler Thromb Vasc Biol. 2000; 20(6):1622-9. [PubMed: 10845881]

31. Persson J, et al. Ultrasound-determined intima-media thickness and atherosclerosis. Direct and indirect validation. Arterioscler Thromb. 1994; 14(2):261-4. [PubMed: 8305418]

32. Li R, et al. B-mode-detected carotid artery plaque in a general population. Atherosclerosis Risk in Communities (ARIC) Study Investigators. Stroke. 1994; 25(12):2377-83. [PubMed: 7974576]

33. Rosfors $\mathrm{S}$, et al. Relationship between intima-media thickness in the common carotid artery and atherosclerosis in the carotid bifurcation. Stroke. 1998; 29(7):1378-82. [PubMed: 9660390]

34. Prati P, et al. Determinants of carotid plaque occurrence. A long-term prospective population study: the San Daniele Project. Cerebrovasc Dis. 2006; 22(5-6):416-22. [PubMed: 16912475]

35. Eigenbrodt ML, et al. B-mode ultrasound common carotid artery intima-media thickness and external diameter: cross-sectional and longitudinal associations with carotid atherosclerosis in a large population sample. Cardiovasc Ultrasound. 2008; 6:10. [PubMed: 18321381]

36. Johnsen SH, et al. Monocyte count is a predictor of novel plaque formation: a 7-year follow-up study of 2610 persons without carotid plaque at baseline the Tromso Study. Stroke. 2005; 36(4): 715-9. [PubMed: 15746459]

37. Markert MS, et al. Ethnic differences in carotid artery diameter and stiffness: the Northern Manhattan Study. Atherosclerosis. 2011; 219(2):827-32. [PubMed: 21906739]

38. Li H, Wong KS. Racial distribution of intracranial and extracranial atherosclerosis. J Clin Neurosci. 2003; 10(1):30-4. [PubMed: 12464517]

39. Spence JD, et al. An approach to ascertain probands with a non-traditional risk factor for carotid atherosclerosis. Atherosclerosis. 1999; 144(2):429-34. [PubMed: 10407504] 
40. Rundek T, et al. Traditional risk factors are not major contributors to the variance in carotid intimamedia thickness. Stroke. 2013; 44(8):2101-8. [PubMed: 23704105]

41. Bis JC, et al. Meta-analysis of genome-wide association studies from the CHARGE consortium identifies common variants associated with carotid intima media thickness and plaque. Nat Genet. 2011; 43(10):940-7. [PubMed: 21909108]

42. Sacco RL, et al. Heritability and linkage analysis for carotid intima-media thickness: the family study of stroke risk and carotid atherosclerosis. Stroke. 2009; 40(7):2307-12. [PubMed: 19498180]

43. Wang L, et al. Fine mapping study reveals novel candidate genes for carotid intima-media thickness in Dominican Republican families. Circ Cardiovasc Genet. 2012; 5(2):234-41. [PubMed: 22423143]

44. Gardener H, et al. Carotid plaque and candidate genes related to inflammation and endothelial function in Hispanics from northern Manhattan. Stroke. 2011; 42(4):889-96. [PubMed: 21393601] 

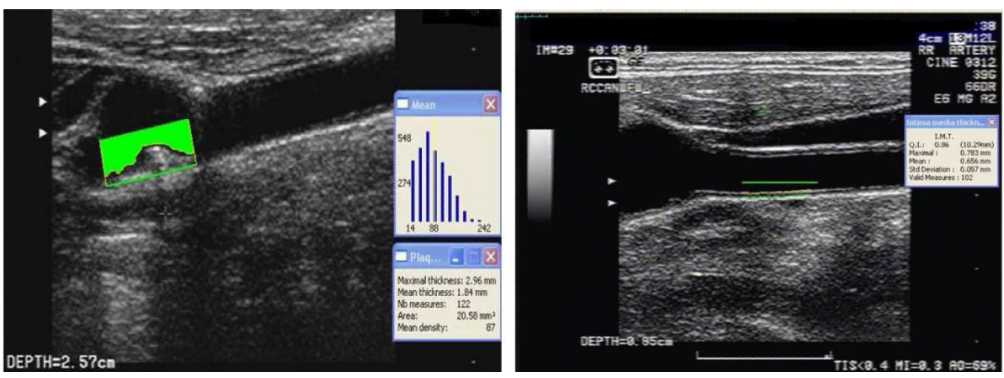

Figure 1.

The left side is a representation of a left bifurcation carotid artery plaque measurement, and the right side is a representation of right common carotid artery cIMT measurement using high-resolution B-model ultrasound. 


\section{Table 1}

Characteristics of the study population

\begin{tabular}{|l|c|c|c|}
\hline & Overall $(\mathbf{N}=\mathbf{1 7 8 7})$ & Plaque $(\mathbf{N = 1 0 2 6})$ & No plaque $(\mathbf{N}=\mathbf{7 6 1})$ \\
\hline Age (years), mean \pm SD & $66 \pm 9$ & $68 \pm 9$ & $63 \pm 8$ \\
\hline Male sex, \% & 40 & 42 & 37 \\
\hline White, \% & 17 & 22 & 12 \\
\hline Black, \% & 19 & 20 & 17 \\
\hline Hispanic, \% & 61 & 56 & 69 \\
\hline Hypertension, \% & 70 & 74 & 65 \\
\hline Diabetes, \% & 19 & 23 & 15 \\
\hline Statin use, \% & 11 & 12 & 10 \\
\hline LDL, mean \pm SD & $128.17 \pm 35.05$ & $129.81 \pm 36.27$ & $125.99 \pm 33.26$ \\
\hline HDL, mean \pm SD & $46.65 \pm 14.43$ & $46.43 \pm 14.42$ & $46.94 \pm 14.46$ \\
\hline Triglycerides, mean \pm SD & $134.79 \pm 79.20$ & $135.90 \pm 75.80$ & $133.31 \pm 83.54$ \\
\hline BMI, mean \pm SD & $28.2 \pm 5.0$ & $28.0 \pm 4.9$ & $28.4 \pm 5.2$ \\
\hline Baseline cIMT (mm), mean \pm SD & $0.92 \pm 0.09$ & $0.94 \pm 0.09$ & $0.90 \pm 0.08$ \\
\hline
\end{tabular}

Abbreviations:

BMI (body mass index), cIMT (carotid intima-media thickness), LDL (Low-density lipoproteins), HDL (High-density lipoproteins) 


\section{Table 2}

Cross-sectional association between carotid IMT, and carotid plaque prevalence, thickness and area at baseline

\begin{tabular}{|c|c|c|c|c|c|}
\hline & $\begin{array}{l}\text { Plaque presence } \\
\text { (N=1026) OR } \\
(95 \% \text { CI })\end{array}$ & $\begin{array}{l}\text { Plaque thickness } \\
(\mathrm{mm}) 50 \% \\
\text { effect, p-value }\end{array}$ & $\begin{array}{l}\text { Plaque thickness } \\
\text { (mm) 75\% } \\
\text { effect, p-value }\end{array}$ & $\begin{array}{l}\text { Plaque area } \\
\left(\mathrm{mm}^{2}\right) 50 \% \\
\text { effect, p-value }\end{array}$ & $\begin{array}{l}\text { Plaque area } \\
\left(\mathrm{mm}^{2}\right) 75 \% \\
\text { effect, p-value }\end{array}$ \\
\hline \multicolumn{6}{|c|}{ cIMT per $0.1 \mathrm{~mm}$} \\
\hline Model 1 & $1.87(1.65-2.12)$ & $0.56,<0.0001$ & $0.32,<0.0001$ & $4.48,<0.0001$ & $9.11,<0.0001$ \\
\hline Model 2 & $1.68(1.47-1.91)$ & $0.46,<0.0001$ & $0.26,<0.0001$ & $3.31,<0.0001$ & $7.21,<0.0001$ \\
\hline Model 3 & $1.72(1.50-1.97)$ & $0.46,<0.0001$ & $0.26,<0.0001$ & $3.45,<0.0001$ & $7.08,<0.0001$ \\
\hline \multicolumn{6}{|c|}{$\begin{array}{l}\text { cIMT in left Bif and ICA per } \\
0.1 \mathrm{~mm} \text { in relation to plaque in } \\
\text { the left Bif and ICA (N=776 } \\
\text { with plaque) }\end{array}$} \\
\hline Model 1 & $1.48(1.34-1.64)$ & $0.25,<0.0001$ & $0.27,<0.0001$ & $0.85,<0.0001$ & $2.85,<0.0001$ \\
\hline Model 2 & $1.41(1.27-1.57)$ & $0.25,<0.0001$ & $0.20,<0.0001$ & $0.85,<0.0001$ & $2.28,<0.0001$ \\
\hline Model 3 & $1.44(1.29-1.60)$ & $0.28,<0.0001$ & $0.23,<0.0001$ & $0.96,<0.0001$ & $2.59,<0.0001$ \\
\hline \multicolumn{6}{|c|}{$\begin{array}{l}\text { cIMT in right Bif and ICA per } \\
0.1 \mathrm{~mm} \text { in relation to plaque in } \\
\text { the right Bif and ICA ( } N=777 \\
\text { with plaque) }\end{array}$} \\
\hline Model 1 & $1.48(1.34-1.63)$ & $0.26,<0.0001$ & $0.23,<0.0001$ & $0.85,<0.0001$ & $2.67,<0.0001$ \\
\hline Model 2 & $1.46(1.31-1.62)$ & $0.26,<0.0001$ & $0.22,<0.0001$ & $0.85,<0.0001$ & $2.45,<0.0001$ \\
\hline Model 3 & $1.46(1.31-1.63)$ & $0.24,<0.0001$ & $0.24,<0.0001$ & $0.93,<0.0001$ & $2.47,<0.0001$ \\
\hline \multicolumn{6}{|c|}{$\begin{array}{l}\text { cIMT in CCA per } 0.1 \mathrm{~mm} \text { in } \\
\text { relation to any plaque }\end{array}$} \\
\hline Model 1 & $1.68(1.52-1.86)$ & $0.52,<0.0001$ & $0.28,<0.0001$ & $3.52,<0.0001$ & $7.07,<0.0001$ \\
\hline Model 2 & $1.46(1.31-1.62)$ & $0.36,<0.0001$ & $0.20,<0.0001$ & $2.35,<0.0001$ & $5.25,<0.0001$ \\
\hline Model 3 & $1.51(1.35-1.69)$ & $0.36,<0.0001$ & $0.18,<0.0001$ & $2.54,<0.0001$ & $4.67,<0.0001$ \\
\hline
\end{tabular}

Model 1: univariate

Model 2: controlling for age, sex, race/ethnicity

Model 3: controlling for age, sex, race/ethnicity, systolic blood pressure, diastolic blood pressure, anti-hypertensive medication use, diabetes, LDL, HDL, triglycerides, BMI, statin use 


\section{Table 3}

Prospective association between IMT at baseline and plaque presence at follow up

\begin{tabular}{|c|c|}
\hline & Incident plaque, OR (95\% CI) \\
\hline cIMT per 0.1mm $\mathbf{( N = 7 6 8 )}$ & N=334 new plaque \\
\hline Model 1 & $1.13(1.02-1.27)$ \\
\hline Model 2 & $1.06(0.95-1.19)$ \\
\hline Model 3 & $1.06(0.94-1.19)$ \\
\hline cIMT per 0.1mm, among those without plaque at baseline (N=324) & N=130 with incident plaque \\
\hline Model 1 & $1.24(1.03-1.50)$ \\
\hline Model 2 & $1.19(0.97-1.46)$ \\
\hline Model 3 & $1.19(0.96-1.48)$ \\
\hline Model 1 & $1.09(0.99-1.20)$ \\
\hline Model 2 & $1.10(0.99-1.21)$ \\
\hline Model 3 & $1.10(1.00-1.22)$ \\
\hline cIMT change from baseline to follow up per 0.1mm (N=768) & \\
\hline Model 1 & $1.08(0.93-1.25)$ \\
\hline Model 2 & $1.08(0.93-1.26)$ \\
\hline Model 3 & $1.07(0.91-1.26)$ \\
\hline
\end{tabular}

Model 1: controlling for time between measurements

Model 2: controlling for time between measurements, age at baseline, sex, race/ethnicity

Model 3: controlling for time between measurements, age at baseline, sex, race/ethnicity, systolic blood pressure, diastolic blood pressure, antihypertensive medication use, diabetes, LDL, HDL, triglycerides, statin use, BMI 


\section{Table 4}

Prospective association between segment-specific IMT at baseline and plaque presence at follow up

\begin{tabular}{|c|c|}
\hline & Incident plaque, OR (95\% CI) \\
\hline cIMT in the CCA per $0.1 \mathrm{~mm}(\mathrm{~N}=768)$ & $\mathrm{N}=334$ new plaque in any segment \\
\hline Model 1 & $1.10(1.01-1.20)$ \\
\hline Model 2 & $1.05(0.96-1.15)$ \\
\hline Model 3 & $1.03(0.94-1.14)$ \\
\hline cIMT in the CCA per $0.1 \mathrm{~mm}$, among those without plaque at baseline $(\mathrm{N}=324)$ & $\mathrm{N}=130$ with incident plaque in any segment \\
\hline Model 1 & $1.16(1.01-1.34)$ \\
\hline Model 2 & $1.13(0.97-1.32)$ \\
\hline Model 3 & $1.14(0.96-1.35)$ \\
\hline Left cIMT in the ICA and Bifurcation per $0.1 \mathrm{~mm}(\mathrm{~N}=768)$ & $\mathrm{N}=161$ new plaque in the left ICA and bifurcation \\
\hline Model 1 & $0.96(0.85-1.08)$ \\
\hline Model 2 & $0.93(0.83-1.05)$ \\
\hline Model 3 & $0.94(0.83-1.06)$ \\
\hline $\begin{array}{l}\text { Left cIMT in the ICA and Bifurcation per } 0.1 \mathrm{~mm} \text {, among those without plaque at } \\
\text { baseline ( } \mathrm{N}=324)\end{array}$ & $\begin{array}{c}\mathrm{N}=100 \text { with incident plaque in the left ICA and } \\
\text { bifurcation }\end{array}$ \\
\hline Model 1 & $1.11(0.93-1.32)$ \\
\hline Model 2 & $1.07(0.89-1.29)$ \\
\hline Model 3 & $1.10(0.90-1.33)$ \\
\hline Right cIMT in the ICA and Bifurcation per $0.1 \mathrm{~mm}(\mathrm{~N}=768)$ & $\begin{array}{l}\mathrm{N}=160 \text { new plaque in the right ICA and } \\
\text { bifurcation }\end{array}$ \\
\hline Model 1 & $0.96(0.86-1.07)$ \\
\hline Model 2 & $0.93(0.83-1.04)$ \\
\hline Model 3 & $0.92(0.81-1.03)$ \\
\hline $\begin{array}{l}\text { Right cIMT in the ICA and Bifurcation per } 0.1 \mathrm{~mm} \text {, among those without plaque at } \\
\text { baseline }(\mathrm{N}=324)\end{array}$ & $\begin{array}{l}\mathrm{N}=100 \text { with incident plaque in the right ICA and } \\
\text { bifurcation }\end{array}$ \\
\hline Model 1 & $1.19(1.00-1.41)$ \\
\hline Model 2 & $1.15(0.96-1.38)$ \\
\hline Model 3 & $1.12(0.93-1.35)$ \\
\hline \multicolumn{2}{|l|}{ CCA cIMT change from baseline to follow up per $0.1 \mathrm{~mm}(\mathrm{~N}=768)$} \\
\hline Model 1 & $1.09(1.01-1.18)$ \\
\hline Model 2 & $1.09(1.00-1.18)$ \\
\hline Model 3 & $1.10(1.01-1.20)$ \\
\hline \multicolumn{2}{|l|}{$\begin{array}{l}\text { CCA cIMT change from baseline to follow up per } 0.1 \mathrm{~mm} \text {, among those without } \\
\text { plaque at baseline ( } N=324)\end{array}$} \\
\hline Model 1 & $1.07(0.95-1.20)$ \\
\hline Model 2 & $1.06(0.94-1.19)$ \\
\hline Model 3 & $1.05(0.92-1.20)$ \\
\hline \multicolumn{2}{|l|}{$\begin{array}{l}\text { Left ICA and bifurcation cIMT change from baseline to follow up per } 0.1 \mathrm{~mm} \\
(\mathrm{~N}=768)\end{array}$} \\
\hline Model 1 & $1.01(0.92-1.10)$ \\
\hline
\end{tabular}




\begin{tabular}{|c|c|}
\hline & Incident plaque, OR (95\% CI) \\
\hline Model 2 & $1.01(0.91-1.10)$ \\
\hline Model 3 & $1.01(0.91-1.11)$ \\
\hline $\begin{array}{l}\text { Left ICA and bifurcation cIMT change from baseline to follow up per 0.1mm, } \\
\text { among those without plaque at baseline (N=324) }\end{array}$ \\
\hline Model 1 & $1.00(0.88-1.15)$ \\
\hline Model 2 & $1.03(0.90-1.19)$ \\
\hline Model 3 & $1.01(0.87-1.18)$ \\
\hline $\begin{array}{l}\text { Right ICA and bifurcation cIMT change from baseline to follow up per 0.1mm } \\
\text { (N=768) }\end{array}$ & \\
\hline Model 1 & $1.05(0.96-1.15)$ \\
\hline Model 2 & $1.05(0.96-1.16)$ \\
\hline Model 3 & $1.06(0.97-1.17)$ \\
\hline $\begin{array}{l}\text { Right ICA and bifurcation cIMT change from baseline to follow up per 0.1mm, } \\
\text { among those without plaque at baseline (N=324) }\end{array}$ & $1.00(0.88-1.15)$ \\
\hline Model 1 & $0.99(0.86-1.14)$ \\
\hline Model 2 & $0.99(0.86-1.15)$ \\
\hline Model 3 & \\
\hline
\end{tabular}

Model 1: controlling for time between measurements

Model 2: controlling for time between measurements, age at baseline, sex, race/ethnicity

Model 3: controlling for time between measurements, age at baseline, sex, race/ethnicity, systolic blood pressure, diastolic blood pressure, antihypertensive medication use, diabetes, LDL, HDL, triglycerides, statin use, BMI 